\title{
The ASTRI Mini-Array: a breakthrough in the Cosmic Ray study
}

\section{Cardillo ${ }^{a}$ for the ASTRI Project*}

${ }^{a}$ IAPS-INAF, Via del Fosso del Cavaliere 100, Rome, Italy

*http://WwW.astri.inaf.it

E-mail: martina.cardillo@inaf.it

Despite the enormous efforts done in very recent years, both theoretically and experimentally, the basic three questions about the CR origin remain without clear answers: what are their sources, how are they accelerated, how do they propagate? Gamma-ray astronomy plays a fundamental role in this field. Both relativistic protons and electrons can emit in the gamma-ray band with different processes but only the detection of hadronic gamma-ray emission is a direct proof of Cosmic-Ray acceleration. Distinguishing leptonic and hadronic components is one of the most tricky issues in the high energy astrophysics, however, a gamma-ray detection at about $100 \mathrm{TeV}$ would be a direct proof of the hadronic origin of the emission. Consequently, not only it would directly confirm the presence of CR acceleration in a source but also it gives us a large amount of information about their sources, their parent protons and their propagation.The ASTRI MiniArray, with its unprecedented sensitivity and angular resolution at $\mathrm{E}>10 \mathrm{TeV}$, will provide a fundamental contribution to close some of the most important CR open issues.

$37^{\text {th }}$ International Cosmic Ray Conference (ICRC 2021)

July 12th - 23rd, 2021

Online - Berlin, Germany 


\section{Introduction}

\subsection{The ASTRI Project: the ASTRI Mini-Array}

During the last decades, a large amount of Very High Energy (VHE) $\gamma$-ray data were collected by the three current Imaging Atmospheric Cherenkov Telescopes (IACTs) H.E.S.S.[1], MAGIC [2] and VERITAS [3] and the more recent Water Cherenkov Detectors (WCD) HAWC [4, 5] and LHAASO [6] added new fundamental information stressing how important are new knowledge and technology in order to understand the VHE universe. In this context, it was conceived the INAF project ASTRI (Astrofisica con Specchi a Tecnologia Replicante Italiana) Mini-Array (ASTRI MA), an array of nine $4 \mathrm{~m}$ double mirror IACTs in the North Hemisphere, at the Observatorio del Teide (Tenerife, Spain). Realized thanks to an INAF commitment with the Italian government and international Partners (University of Sao Paulo/FPESP - Brazil, North-West University - South Africa, IAC (Instituto de Astrofisica de Canarias) - Spain), it has more than 150 researchers working on it $[7,8]$.

ASTRI MA will operate between $0.1 \mathrm{TeV}$ and $200 \mathrm{TeV}$ and every telescope is a copy of the dualmirror Schwarzschild-Coudé (SC) ASTRI-Horn telescope. This is one of the four different end-toend prototypes for the Small Size Telescopes (SSTs) in the South Site of the Cherenkov Telescope Array Observatory (CTAO) [9], developed within the ASTRI Project [10-12]; its structure and mirrors were selected for the implementation in the SSTs of CTAO.

\subsection{The ASTRI Mini-Array: Performances and Science}

The first telescope of ASTRI MA will be ready within the first half of 2022, other two will be added for the end of 2022 and the whole project for the Spring of 2024. Consequently, ASTRI MA will operate some years before the completion of CTAO; the high-energy universe could be unveiled in the very near future. Indeed, ASTRI MA will have unprecedented performances [13] and it will add new data and information at the huge work done up to now in the VHE band [14, 15].

One of the most important characteristic of the ASTRI MA is the very large Field of View (FoV) of about $10^{\circ}$ with an almost homogeneous off-axis acceptance up to about 3 degree (and within a factor of 2 up to 5 degree). This will allow us to observe and analyze multi-target fields and extended sources without performance-losses. Moreover, we will be able to do surveys and the chance for serendipitous discoveries will be enhanced. The large field analysis will be supported by the a very high sensitivity $\left(\approx 10^{-12} \mathrm{erg} / \mathrm{cm}^{-2} / \mathrm{s}\right.$ at $\left.10 \mathrm{TeV}\right)$ and enhanced energy and angular resolution ( $\leq 10 \%$ and $\leq 0.1^{\circ}$ at $10 \mathrm{TeV}$ ) [13]. We will study faint sources and will add important spectral and morphological constraints on detected VHE sources.

In the last year, we simulated ASTRI MAevents for some selected $\gamma$-ray sources, analysing them in order to show what we expect for the future. We used the Instrument Response Functions (IRFs) produced from dedicated Monte Carlo simulation and they include all information about the array parameters [13]. For simulation and analysis we used both the external tools used in the CTA Consortium, CTOOLS [16] and Gammapy [17]. These simulations show that the ASTRI MA will be able to deeply understanding issues as like as the origin of Cosmic Rays (CRs), the characteristics of the Ultra High Energy CRs (UHECRs), the Extragalactic Background Light (EBL) nature, fundamental physics problems until the multi-messangers astrophysics. A detailed paper about that is in progress $[14,15]$ but here we focus on one particular topic: the galactic CR origin. 


\section{The ASTRI MA and the Cosmic-Ray origin}

Despite their discovery was in 1912 and the large amount of data collected up to now, CR origin is still an open issue. The most convincing theoretical scenario, at least for Galactic CRs (GCRs), is the Diffusive Shock Acceleration (DSA) mechanism and the initial paradigm considered the Supernova Remnants (SNRs) their most probable sources. However, experimental data have opened new hypothesis and changed some theoretical paradigms [18-21, for recent reviews]

At the lowest energies, we have the proof of CR energization from some middle-aged SNRs as W44 [22-24], IC443 [24] and W51c [25]. However, we have no certainty of the presence of freshly accelerated CRs. The main hypothesis for their hadronic $\gamma$-ray emission are reacceleration of pre-existent CRs [26, 27] or suppression of diffusion coefficient close to the sources [28, 29].

At the highest energies, we have no sure $\gamma$-ray detection of SNRs above $100 \mathrm{TeV}$ (proof of possible Pevatron nature) and this could depend on a detection problem. Indeed, taking into account the non-resonant Bell instability [30], only Type II SNRs can accelerate particles above $1 \mathrm{PeV}$ and only in the first 100 years of their life [31]. Consequently, we do not know SNRs quite young in order to be in their pevatron phase. The only hope that we have are Molecular Clouds (MCs) because CRs escaped from sources can be trapped inside them, emitting radiation in the VHE band of the $\gamma$-ray spectrum [32]. But there is another chance: SNRs could not be the only sources of GCRs. In the last years, $\gamma$-ray emission was detected at about or above $100 \mathrm{TeV}$ from different kind of sources: Galactic Center region [33, 34], Superbubbles [34, 35] and Pulsar Wind Nebulae, with the Crab Nebula as the most important example [36-38]

The ASTRI MA will clarify important CR open issues, improving spectral resolution of some candidates Pevatrons, constraining their spectral parameters and resolving their morphology in such a way to distinguish different origin for the $\gamma$-ray emission. All the details about this work will be deeply described in [14].

\subsubsection{Candidate Pevatrons}
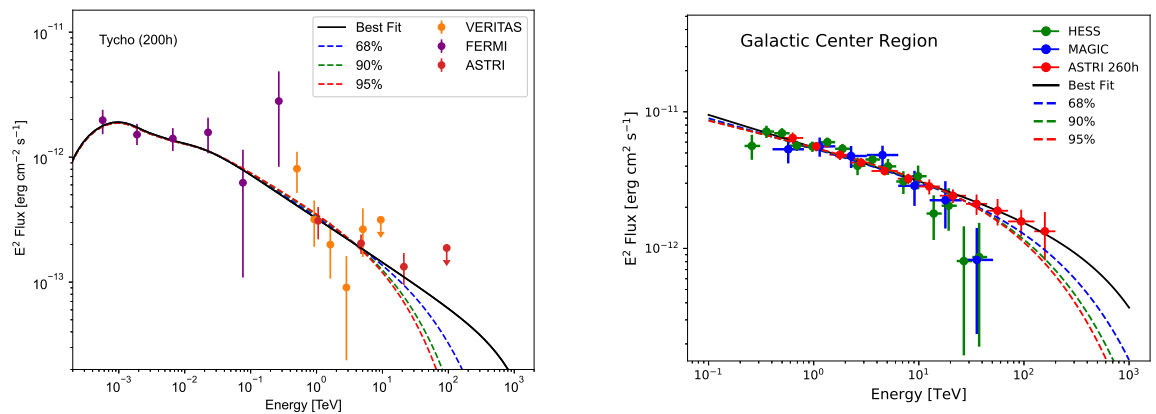

Figure 1: Left: 200 hours of observations of Tycho SNR. The $\gamma$-ray data from Fermi-LAT (84 months) and VERITAS (147 hr) together with the ASTRI MA simulations (red). The dashed lines show the PL fit with cut-off energies of 0.29, 0.41, 1.27 PeV. Right: GC region - H.E.S.S., MAGIC and ASTRI Mini-Array (260 hr) spectra fitted with a proton population with a best fit cut-off at $120 \mathrm{PeV}$ (black solid line). The blue, green and red solid lines indicates cut-off energies, 3.50 PeV, 2.0 PeV and 1.7 PeV. 
The first breakthrough of the ASTRI MA is the chance to detect $100 \mathrm{TeV} \gamma$-ray emission from possible candidate pevatron sources. We studied the case of the Tycho SNR. This very faint $\gamma$-ray source is one of the youngest SNRs, detected at VHE by VERITAS [39] but these data are not sufficient to exclude the presence of a cut-off. Starting from the VERITAS spectral model, our simulations show that with only 200 hours of exposure, ASTRI MA can exclude the presence of a cut-off below $100 \mathrm{TeV}$ if Tycho is a real Pevatron (Fig.1, left). Moreover, it will be able to solve its morphology, allow us to understand possible multi-wavelength emission correlations.

Important improvement there will be also in the case of the GC region, recently detected by H.E.S.S., VERITAS [40] and MAGIC [37] up to $50 \mathrm{TeV}$ without evidence of a cut-off. With the same exposure time of H.E.S.S., 260 hours, ASTRI MA will be able to map the whole GC region with a single observation and to detect, if present, its $100 \mathrm{TeV}$ emission (see Fig. 1, right). The very good angular resolution will solve several sources within the region, confirming or disproving the current hypothesis about the VHE $\gamma$-ray emission [34].

After the last papers by HAWC and LHAASO collaborations [35, 38], the number of detected sources in the VHE band is strongly increased. Our simulation show how the ASTRI MA contribution will be fundamental for some of them. We considered two examples.

The first one is the source eHWC J1907+368. Using the morphology from VERITAS [42] and the power law spectrum from HAWC [4], simulations show that we can detect possible pevatron emission with only 100 hours of exposure, constraining the presence of a cut-off at $1.6 \mathrm{PeV}$ with 95\% of confidence (50 TeV without ASTRI MA, Fig.2, left). Moreover, thanks to its unprecedented angular resolution, the ASTRI MA can identify correlated counterpart allowing to understand if the $\gamma$-ray emission is leptonic, due to PWN, or hadronic, related to CR particle acceleration (Fig.2, center) [43].
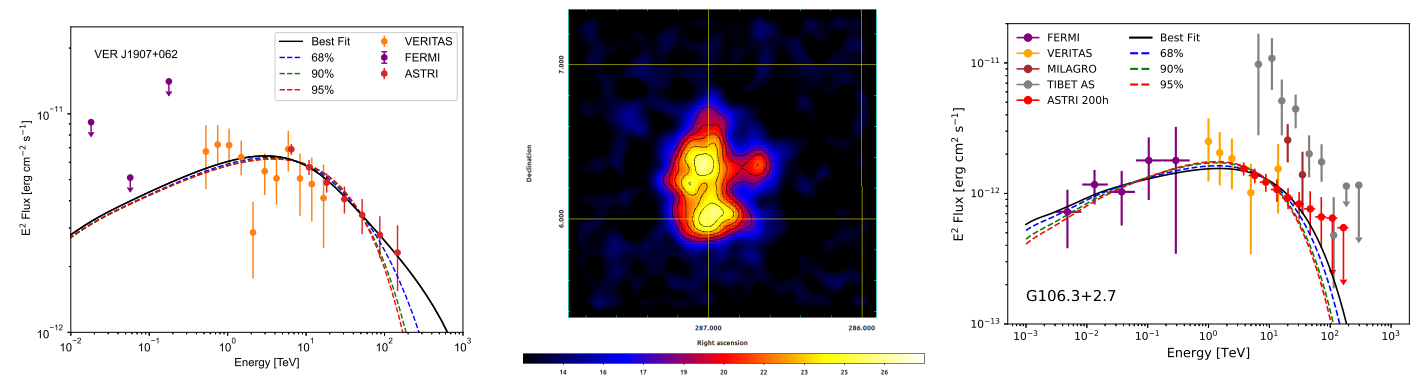

Figure 2: Left: eHWC J1907+368 - $\gamma$-ray data from Fermi-LAT [44], VERITAS and ASTRI MA simulations (100h). The blue, green and red lines show the broken power law fit with a cut-off energy of 1.67, 0.54 and 0.4 PeV Center: eHWC J1907+368 - ASTRI MAsimulated counts map (200 h). Right: G106.7+2.7 SNR $\gamma$-ray data from Fermi-LAT [45], VERITAS and ASTRI MA (200 h). Milagro and Tibet AS data points are shown for reference only. The solid lines show the emission from a proton population with a best-fit cut-off energy of $350 \mathrm{TeV}$ (black line) and lower-limit energy of 250 (blue line), 180 (green line) and 160 (red line) $\mathrm{TeV}$.

This ambiguity is present also in the second treated example, the SNR G106.7+2.7, detected at TeV energies by VERITAS [46], MILAGRO [47, 48], HAWC [35] and Tibet AS $\gamma$ [49]. The ASTRI MA angular resolution will confirm a possible association between its $\gamma$-ray emission and a near MC, identifying possible different morphologies at different energies. As shown in Fig.2 (right), 
taking into account the morphology and spectrum from VERITAS, the ASTRI MA will extend the SNR spectrum up to $100 \mathrm{TeV}$ and will constrain the cut-off at energies of about $500 \mathrm{TeV}$ with the $69 \%$ of confidence.

\subsubsection{Cosmic Ray Propagation}

Even if most of detected SNRs passed their possible pevatron phase, MCs can help us to identify which of them were Pevatrons in the past. Moreover, CR trapped within MCs retain information about the $\mathrm{CR}$ escape process and, consequently, the diffusion coefficient value in the source surroundings. A deeper understanding of diffusion can also solve the ambiguity of the middle aged SNR spectra, re-acceleration or diffusion suppression.

In this context, we have simulated and analyzed with ASTRI MA two famous middle-aged SNRs, $\gamma$-Cygni [50] and W28 [51]. Both these sources emit VHE $\gamma$-ray emission and have MCs close to them. We will be able to solve their emission and to constraint the diffusion parameters. These constraints will come also from the spectral study of the sources because ASTRI MA will add some high energy spectral point that can solve the ambiguity between different diffusion models.

For $\gamma$-Cygni, our results points toward the "Model B" [14] that considers the diffusion coefficient suppression near the source (see Fig.3, left). For W28, we focused our analysis on the most luminous of the three TeV MCs in its surrounding, HESS J1800-240B, in order to understand the energy dependence of $D(E) \propto E^{s}$. Testing the two extreme cases of diffusion regime, Kolmogorov$\left(D(E) \propto E^{0.35}\right)$ and Kraichnan-like $\left(D(E) \propto E^{0.5}\right)$ turbulent spectrum, from our simulations it is evident that ASTRI MA will be fundamental in order to identify the correct behaivor.
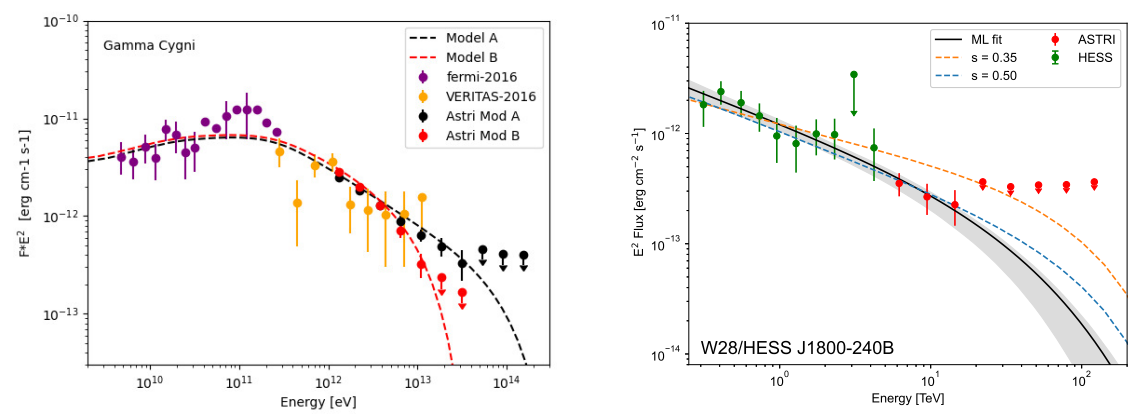

Figure 3: Left: $\gamma$-ray spectrum of $\gamma$-Cygni. Data from Fermi-LAT [52] and VERITAS while theoretical models A and B are showed with dashed curves. Black and red dots show the ASTRI MA simulations for model A and B, respectively (200 h). Right: $\gamma$-ray spectrum of HESS J1800-240B. Data are from H.E.S.S. (green dots) and ASTRI MA simulations (200 h, red dots) for Kolmogorov turbulence model (blue dashed line). The orange dashed line shows the predicted Kraichnan-like spectrum and the best fit is represented by the solid black line.

\subsubsection{ASTRI MA and hadrons/leptons ambiguity}

In the last years, the Crab nebula was unexpectedly detected above $100 \mathrm{TeV}[36-38,53]$. According to theory, PWN emit leptonic $\gamma$-ray that cannot reach these energies because of the Klein-Nishina suppression of the Compton cross section. Consequently, this detection is very puzzling and leads naturally to consider two different hypothesis; PWN can accelerate leptons up 
to $100 \mathrm{TeV}$ or PWN $\gamma$-ray emission is dominated by hadrons at the highest energies [54]. This new context is supported also by new PWNae detected above $100 \mathrm{TeV}$ by HAWC [35] and LHAASO [38].

In the case of Crab, we tested two different wind models with different amount of protons and different values of the Lorentz factors. Despite the presence of VHE points by LHAASO, the ASTRI MA contribution above $200 \mathrm{TeV}$ will add crucial spectral point that allow us to clearly recognize a possible hadronic contribution (see Fig.4, top). In the last weeks, LHAASO collaboration published another paper on Science with a Crab detection at PeV energies [55]. We had no time to consider these last results in our work but we want to stress that the ASTRI MA angular resolution will be really crucial in order to solve $\gamma$-ray emission from this tricky source and to finally understand the origin of this huge amount of energy.

Moreover, ASTRI MA will have a fundamental role in the study and understanding of the extended TeV halos recently detected around some PWNes [5, 56]. These halos could give critical information about particle escape efficiency from PWN and their transport in its surroundings, with direct implication on the positron excess issue [57-60]. We tested the case of Geminga, finding out that ASTRI MA will solve a large fraction of its TeV halo thanks to the large FoV and the very good angular resolution, allowing a good resolution reconstruction of its spectrum and radial profile.

\subsubsection{Ultra High Energy Cosmic Rays}

We want to briefly mention also the important contribution that ASTRI MA will have in the understanding of UHECRs acceleration. Starburst Galaxies are one of the main candidates for their origin because of their intense Star Formation Rate (SFR) and extreme propertiesof the Interstellar Medium (ISM) [61]. Despite our increased knowledge about this context in the last decade, we have no chances to constrain the maximum energy reached by these sources and, consequently, to confirm their hadronic emission.

ASTRI MA will reach energies above few TeV also in the case of Starburst Galaxies as showed in our chosen source, M82, our the best candidate (see Fig.4, bottom). we can fix the cut-off energies, understanding the $\gamma$-ray absorption and the extent of the contribution of Starburst galaxies to the measured diffuse neutrino flux.

\section{Conclusions and Future}

The ASTRI MA will be the precursor of the CTAO, ready to start operations in 2024 with the first three telescopes ready for the end of the next year. Our first simulations with good but still improvable IRFs show how ASTRI MA will contribute in a fundamental way to the CR context thanks to its unprecedented performances.

We plan to have on average 1000 hours per years available for observations, taking into account bad weather conditions, maintenance operations and the typical canarian "calima". We can reach high zenit angle observations $\left(\leq 60^{\circ}\right)$, necessary for sources like the GC region, and we will able to operate with the presence of the Moon in its "quarter phase" thanks to the SiPM ASTRI camera and to a good developed model of Night Sky Background (NSB) [13]. In this way, we will have all the chosen sources well distributed during the year. The ASTRI MA has all the potentialities to answer the question "What are the sources of Galactic CRs?" in the very near future. 

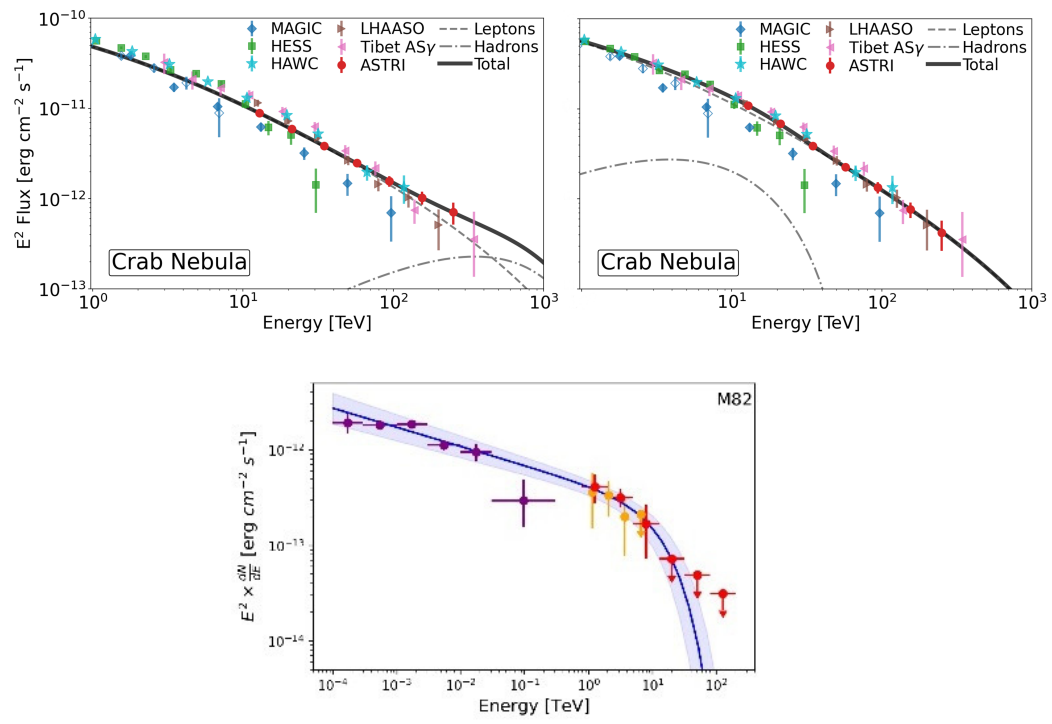

Figure 4: Top Left: ASTRI MA simulated spectrum (500 hours) of the Crab Nebula modelled with a more consistent hadronic component. Top Right: simulated spectrum with a lower hadronic contribution. Bottom: $\gamma$-ray spectrum of M82. Data are from Fermi 4FGLDR2 (purple points, [62], from VERITAS (orange points, [63]), and from ASTRI MA simulations (100 h, red points). The best fit is represented by the solid blue line.

\section{References}

[1] Aharonian, F.A. et al., A\&A457, 899 (2006)

[2] Aleksic, J. et al., Astrop. Phys. 35, 435 (2012)

[3] Weekes, T.C. et al., Astrop. Phys. 17, 221 (2002)

[4] Abeysekara, A.U. et al., ApJ 843, 39 (2017a)

[5] Abeysekara, A.U. et al., ApJ 843, 40 (2017b)

[6] Cao, Z., Chin. Phys. C 34 (2), 249-252 (2010)

[7] Antonelli, L., A., Proc.37th ICRC (2021)

[8] Vercellone, S. et al., Proc.AIPC, 1505, 749 (2012)

[9] Cherenkov Telescope Array Consortium, Science with the Cherenkov Telescope Array, (2019)

[10] Pareschi, G. et al., Proc. 33th ICRC, 33, 1151P (2013)

[11] Giro, E. et al., Proc. SPIE 11119, 11119E (2019)

[12] Scuderi, S. et al., Proc. EPJWC, 209,01001(2019)

[13] Lombardi, S. et al., Proc.37th ICRC (2021)

[14] Vercellone, S. et al., submitted

[15] Vercellone, S. et al., Proc.37th ICRC (2021)

[16] Knodlseder, J. et al., å593, A1 (2016)

[17] Deil, C. et al., Proc. 35th ICRC, 301, 766 (2017)

[18] Blasi, P., AApR 21, 70 (2013)

[19] Amato, E., IJMP, 23, 1430013 (2014)

[20] Gabici, S. et al., IJMPD, 28, 1930022-339 (2019)

[21] Blasi, P., Nuovo Cimento Rivista Serie 42, 549 (2019) 
[22] Giuliani, A. et al., ApJL 742, L30 (2011)

[23] Cardillo, M. et al., A\&A565, A74 (2014)

[24] Ackermann, M. et al., Science 339, 807 (2013)

[25] Jogler, T. and Funk, S., ApJ 816, 100 (2016)

[26] Cardillo, M. et al., å595, A58 (2016)

[27] Cardillo, M., Galaxies7, 49 (2019)

[28] Celli, S. et al., MNRAS 396, 1629 (2009)

[29] Mitchell, A.M.W. et al., MNRAS 503, 3522 (2021)

[30] Schure, K.M. et al., MNRAS 437, 2802 (2014)

[31] Cardillo, M. et al., Astrop. Phys. 69, 1 (2015)

[32] Cristofari, P. et al., Astrop. Phys. 123, 102492 (2020)

[33] H.E.S.S.collaboration, Nature 531, 476 (2016)

[34] H.E.S.S.collaboration, å612, A9 (2018)

[35] HAWC collaboration, Phys.Rev.Lett. 124, 021102 (2020)

[36] Amenomori, M. et al., PRL 123, 051101 (2019)

[37] MAGIC Collaboration, å642, A190 (2020)

[38] Cao, Z. et al., Nature 534, 33 (2021)

[39] Archambault, S. et al., ApJ 836, 23 (2017)

[40] Archer, A. et al., ApJ 821, 129 (2016)

[41] Ahnen, M.L. et al., å601, A33 (2017)

[42] Aliu, E. et al., ApJ 787, 166 (2014)

[43] Crestan, S. et al., MNRAS 505, 2309 (2021)

[44] Acero, F. et al., ApJ 773, 77 (2013)

[45] Xin, Y. et al., ApJ 885, 162 (2019)

[46] Acciari, V.A. et al., ApJL 703, L6 (2009)

[47] Abdo, A.A. et al., ApJL 664, L91 (2007)

[48] Abdo, A.A. et al., ApJL 700, L127(2009)

[49] Tibet AS $\gamma$ Collaboration, Nature Astronomy (2021)

[50] MAGIC Collaboration, NAture 575, 455 (2019)

[51] Aharonian, F.A. et al., å481, 401 (2008)

[52] Fraija, N. et al., ApJ 826, 31 (2016)

[53] HAWC collaboration, arXiv e-prints arXiv:1909.08609 (2019)

[54] Amato, E. et al., å402, 827 (2003)

[55] LHAASO collaboration, Science 373, 425

[56] Linden, T. et al. PRD 96, 103016 (2017)

[57] Evoli, C. et al., PRD 98, 063017 (2018)

[58] Fang, K. et al., ApJ 884, 124 (2019)

[59] Lopez-Coto, R. et al., MNRAS 479, 4526 (2018)

[60] Olmi, B. et al., MNRAS 490, 3608 (2019)

[61] Anchordoqui, L.A. et al., PRD 60, 103001 (1999)

[62] Ballet, J. et al., arXiv e-prints arXiv:2005.11208 (2020), 2005.11208

[63] VERITAS collaboration, Nature 462, 770 (2009) 